\section{Liver in Crohn's Disease}

Though distinctions between the clinical and pathological features of Crohn's disease and ulcerative colitis are often well marked, in some cases they are minimal, and the correct diagnosis is difficult to reach. Of the organs secondarily damaged by ulcerative colitis the liver is so fairly commonly. ${ }^{1}$ In contrast, pathological changes and disordered function of the liver are usually thought to be rare in Crohn's disease. With the tendency for these two diseases to overlap each other both clinically and pathologically it is interesting to study a recent review of Crohn's disease from Birmingham $^{2}$ in which a careful examination of liver structure and function was made and compared with the situation in ulcerative colitis.

One hundred patients with Crohn's disease were studied, of which the majority (65) had evidence of disease in both small and large bowel. Liver function tests were abnormal in one respect or another in up to $23 \%$ of the patients, the most consistently abnormal test being serum albumin. This is obviously not an expression of impaired liver function alone. Nine patients, however, had abnormal bromsulphalein retention at 45 minutes (greater than $10 \%$ ), and all but one of these patients had chronic Crohn's disease of more than ten years' duration. Histological evidence of abnormal liver structure was more commonly found, and the four histological abnormalities- "inflammatory cellular infiltration," "bile duct proliferation," "fatty infiltration," and "Kupffer cell prominence"-were each seen in $40 \%$ or more of the patients studied histologically. In 47 of 49 biopsies $(94 \%)$ there was some histological abnormality, though there was little relationship to the clinical picture and rarely evidence of any serious liver disease. There were no cases of cirrhosis, for example, and only one of amyloid disease.

The same authors compared their findings with those they have reported in ulcerative colitis. ${ }^{3}$ The incidence of histological abnormality was found to be roughly the same, but evidence of the more severe kinds of liver disease was more common in ulcerative colitis. In this series ${ }^{3}$ Crohn's disease was a more potent cause of hepatic damage when it was in the colon than when it was in the small intestine, and a point of interest in the Birmingham series is that serious hepatic disease is likely to be a feature of total colonic Crohn's disease rather than of small-bowel and partial colonic disease.

With these conclusions in mind it is worth studying a similar report from Oxford. A. D. Perrett and colleagues 4 showed that in 100 patients with Crohn's disease biochemical evidence of liver dysfunction based on liver function tests was found in $26 \%$, and here the bromsulphalein test was the one most commonly abnormal. Of those patients who had liver biopsies $50 \%$ showed some histological abnormality, most commonly pericholangitis and focal necrosis or fatty change, but little serious liver disease was found (one case of chronic active hepatitis and one with micronodular cirrhosis). On the other hand in ulcerative colitis 300 patients $^{5}(15 \%)$ showed abnormal liver function tests, and about $60 \%$ of those patients with ulcerative colitis who were examined by biopsy showed abnormal histology. The commonest pathological changes were fatty change in $38 \%$ and pericholangitis in $30 \%$. Eight patients $(16 \%)$ had serious liver disease-chronic active hepatitis, cirrhosis, or amyloidosis.

Though the overall incidence of liver disease is much the same in these two disorders, the pattern is different, with the more serious types of liver disease tending to complicate ulcerative colitis rather than Crohn's disease. Other examples of serious liver disease occasionally found in ulcerative colitis include bile duct carcinoma, ${ }^{67}$ though this was not found in the Birmingham series.

Another important difference seems to concern the presence of immunological abnormalities in the serum in the two diseases. Positive immunofluorescent tests against human colon and a variety of other antibodies were found five to six times more frequently in patients with ulcerative colitis than in Crohn's disease. A similar difference was noted in the level of IgM immunoglobulins, high levels being a feature of ulcerative colitis rather than Crohn's disease.

These facts still leave us in a dilemma, for though liver disease in ulcerative colitis seems to be related to the extent of the colonic lesion, the Oxford workers, unlike their colleagues in Birmingham, did not find a relationship between involvement of the colon by Crohn's disease and hepatic disease. The precise cause of the liver lesions in these two disorders is also unknown. Viral hepatitis and drugs seem not to be important, and the possibility of immunological liver disease in ulcerative colitis is still unproved. The often quoted work of B. N. Brooke and colleagues ${ }^{89}$ suggesting that portal bacteraemia may be an important cause of liver disease, at least in colitis, is criticized by the Oxford workers as lacking controlled observations for comparison. Certainly these workers found that liver biopsies, both in Crohn's disease and in ulcerative colitis, were uniformly sterile on bacterial culture, and a search for bacterial $L$ forms was also unrewarding. In the midst of these unknowns they suggest, in view of the overall similar incidence of liver disorders, both in frequency and in type, found in association with the two conditions, that ulcerative colitis and Crohn's disease may in fact represent different manifestations of a single disorder, and that the liver disease is merely a secondary consequence of chronic inflammatory disease of the intestines, irrespective of its exact type. Pathologists, at least, faced by the perplexing problem of whether a case falls precisely into the diagnostic category of Crohn's disease or ulcerative colitis, will take heart from these suggestions.

1 Pollard, H. M., and Black, M., Archives of Internal Medicine, 1948, 82, 159. 2 Eade, M. N., Cooke, W. T., and Williams, J. A., Scandinavian fournal of Gastroenterology, 1971, 6, 199.

3 Eade, M. N., Annals of Internal Medicine, 1970, 72, 475.

4 Perrett, A. D., et al., Quarterly fournal of Medicine, 1971, 40, 187.

Perrett, A. D., et al., Quarterly fournal of Medicine, 1971, 40, 211.

' Mistilis, S. P., Skyring, A. P., and Goulston, S. J. M., Annals of Internal Medicine, 1965, 63, 17 .

Brooke, B. N., and Slaney, G., Lancet, 1958, 1, 1206.

\section{The Handicapped : Who Cares?}

Though most doctors are aware of the considerable numbers of people who suffer in varying degrees from some form of physical, mental, or sensory impairment, the care of them deserves more thought than it has received. As a starting point the painstaking survey of the handicapped and impaired ${ }^{1}$ by Amelia I. Harris and her colleagues in the Social Survey Division of the Office of Population Censuses and Surveys is worthy of careful study by everyone responsible for community health services.

Just over three million people aged 16 and over with some 
physical, mental, or sensory impairment live in private households in Great Britain. There are age, sex, and regional differences in their frequency distribution. More than half of them are elderly, and the highest numbers of impaired men are in the North of England and in Wales, probably because of the industrial hazards in those parts of the country. The greatest single cause of impairment is arthritis, which affects 700,000 women and 200,000 men. More men than women suffer from coronary disease and lung diseases, also presumably partly owing to their greater exposure to industrial conditions.

Impairment does not always result in the person being handicapped, and most of them can carry out their everyday activities without any or with only minor difficulty. But the survey estimates that there are $1,130,000$ persons $(760,000$ women and 370,000 men) who need support of some kind. Of these $70 \%$ are 65 or over and only $2 \%$ under 30 . One in five handicapped persons is living alone, and few have the use of a telephone to call for help. Of the very severely handicapped needing considerable help some 300 men are living alone, and 8,000 women, the majority being over 74 years of age. The estimate of those classified in the survey as severely handicapped is ten times as great, including some 50,000 women aged 75 and over who are living alone.

All this creates a vast volume of work for general practitioners and the health and social services. Half of the very severely and severely handicapped are seen regularly, usually once a month, by their general practitioners. The elderly in the North were found to be almost twice as likely to have regular visits from their doctors as those in Greater London. The health and social services provide most support through chiropody, home help, and, particularly for the very severely handicapped, the home nursing service. District nurses visit nearly two in every three severely handicapped persons needing day and night care, the remainder being looked after by relatives and friends.

This survey illustrates clearly the great burden of responsibility falling on the doctors and nurses caring for the handicapped and the large volume of work they present to the social services. Clearly the medical factors both in the assessment of these people and in advising on the home support they require are dominant and need to be carefully planned. Here is a superb challenge for the new community physician.

Office of Population Census and Surveys Social Survey Division, Handicapped and Impaired in Great Britain, Parts 1 and 2. London, H.M.S.O., 1971 .

\section{The Lungs in Ankylosing Spondylitis}

Ankylosing spondylitis is now firmly established as a specific systemic disorder. It differs from rheumatoid arthritis in several respects. It mainly affects males, the sacro-iliac joints and spine are regularly involved, but the hands and feet usually escape. Iridocyclitis and cardiac disease are comparatively more common. Subcutaneous nodules are rare, and tests for rheumatoid factor are positive in less than $15 \%$ of cases. During the past decade ankylosing spondylitis has frequently been noted in association with ulcerative colitis, regional enteritis, and psoriasis, and has occasionally been linked with Reiter's syndrome and juvenile rheumatoid arthritis. Reports from Canada and Australia now suggest that the lungs may also be involved in the primary pathological process.

In 1949 K. A. Hamilton ${ }^{1}$ described two patients with chronic infiltrative and fibrotic changes in the upper lobes of the lungs. Descriptions of six similar patients followed in $1965^{2}$ and a further seven in $1968 .{ }^{3}$ One more was added in $1971,{ }^{4}$ giving a total of 16 . Their clinical features were strikingly similar. All had developed spinal ankylosing spondylitis in early adult life. Cough, sputum, and dyspnoea usually started in about the fifth decade, the sputum sometimes becoming profuse. Serial radiographs showed diffuse and almost always bilateral mottling in the upper halves of the lung fields. The mottling progressively increased in size and extent over several years, leading eventually to widesnread fibrosis and cyst formation in the upper lobes, at which time repeated haemoptyses were common. The appearances were similar to those seen in pulmonary tuberculosis, and indeed most of the patients found their way into sanatoria, but tests for tubercle bacilli were consistently negative. They were quite dissimilar to the radiological changes seen in the lungs of some patients with rheumatoid arthritis.

From the evidence of biopsy and post-mortem material the early changes were seen to consist of a patchy pneumonia with round-cell and fibroblastic infiltration, progressing to extensive interalveolar fibrosis with areas of hyalinization. Later, dense pleural and pulmonary fibrosis caused contracture of the lung substance, with the development of many cysts, some of which were large. The changes resembled the fibrosing inflammation which sometimes occurs in the aorta of patients with ankylosing spondylitis.

Evidence that the lungs may be specifically affected in ankylosing spondylitis now seems to be convincing. From such case reports as are available the differential diagnosis seems to have been carefully considered, at least from the respiratory point of view. Tuberculosis, sarcoidosis, pneumonoconiosis, radiation pneumonitis, and syphilis cannot be seriously entertained as alternatives. Fungi have been found in the later stages, but they were almost certainly secondary invaders of bronchiectasis and cysts within the masses of fibrous tissue. The pathological features were unlike those found in fibrosing alveolitis. None of the patients was submitted to the battery of immunological tests that are now available, but there is no reason to suppose that such tests would have been any more enlightening than they are in patients having ankylosing spondylitis without pulmonary involvement.

The frequency with which specific infiltration and fibrosis develop in the lungs remains uncertain, but it must be rare. W. M. Court Brown and (Sir) Richard Doll ${ }^{5}$ analysed the causes of death in an exceptionally large series of patients with ankylosing spondylitis. They noted a $2 \cdot 5$ to 3 times excess of deaths from respiratory causes compared with expected rates, principally from tuberculosis and pneumonia. They considered that their figures "might include a small number due to a type of upper lobe fibrosis" described here. Two extensive clinical studies of ankylosing spondylitis 67 disclosed no instance of specific pulmonary changes nor any evidence of a special predisposition to pulmonary tuberculosis or other respiratory infection.

The question of treatment remains unresolved. Up to the present most patients have been given prolonged courses of antituberculosis drugs without benefit, and little more. The standard remedies for ankylosing spondylitis-namely, acetyl salicylic acid, indomethacin, and phenylbutazone-seem to have no measurable effect on the lungs. Corticosteroid drugs, which are ordinarily reserved for iridocyclitis and the rare 